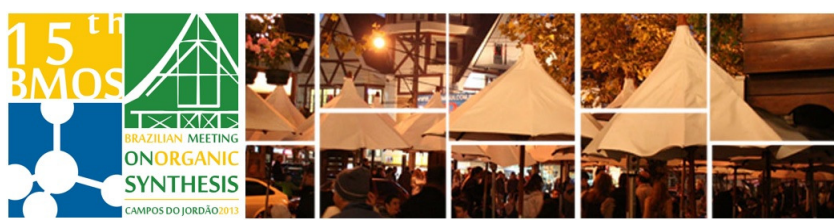

\title{
3H-1,2-dithiole-3-thione to (E)-3-[1-(alkylthio)alkylidene]-3H-1,2- dithiole transformation: theoretical and experimental studies
}

\section{Marcos Couto ${ }^{\text {a, }}$, Mauricio Cabrera ${ }^{a}$, Gustavo A. Echeverría ${ }^{b}$, Oscar E. Piro ${ }^{b}$, Mercedes González a and Hugo Cerecetto ${ }^{\mathrm{a}}$.}

\author{
a Grupo de Química Medicinal, Facultad de Ciencias-Facultad de Química, UdelaR, Iguá 4225, Montevideo, \\ Uruguay. ${ }^{b}$ Dpto. de Física, Facultad de Ciencias Exactas, Universidad Nacional de la Plata Argentina. \\ *mcoutosire@gmail.com
}

Keywords: 3H-1,2-dithiole-3-thione,(E)-3-alkylidene-3H-1,2-dithiole, HSAB theory.

\section{INTRODUCTION}

During the development of hybrid chemopreventive agents containing chalcone framework and $3 \mathrm{H}-1,2-$ dithiole-3-thione moiety an unexpected product was observed. When ADT-OH (Table 1) was subjected to classical alkylation conditions ${ }^{1}$, working in an excess of $\mathrm{KI}$ and $\mathrm{K}_{2} \mathrm{CO}_{3}$, we have obtained the resulting product of the electrophile character of the thiocarbonyl moiety on the 3-position of the 1,2dithiole, instead of the expected nucleophilic-reaction product. The aim of this work was rationalized this observed reactivity from an experimental and theoretical point of view.

\section{RESULTS AND DISCUSSION}

Different conditions were assayed to test the formation of (E)-3-[1-(alkylthio)alkylidene]-3H-1,2dithiole. Experiments consisted on studying the relevance of the reactants, i.e. $\mathrm{KI}$, enolates, and $3 \mathrm{H}$ 1,2-dithiole-3-thiones combined with microwave assisted synthesis. Firstly, we analysed the generation of product 2 (Table 1), from ADT-OH, in absence of $\mathrm{KI}$ and the reactivity of $3 \mathrm{H}-1,2$-dithiole-3thiones with other enolates different of $\alpha$ chloroacetone to explore the 3-thiocarbonyl electrophile. The results (Table 1) showed that the incorporation of acetyl-moieties in product $\mathbf{2}$ appeared when was used a-chloroacetone as enolate and in the presence of $\mathrm{KI}$.

Table 1. Reaction conditions to generate $\mathbf{1}$ and 2 .

\begin{tabular}{|c|c|c|c|c|c|}
\hline \multicolumn{2}{|c|}{ ADT-OH } & 2 & $0=$ & 1 & \\
\hline \multirow{2}{*}{ Run } & \multirow[b]{2}{*}{ KI } & \multirow[b]{2}{*}{ Base } & \multirow{2}{*}{ methylketone } & \multicolumn{2}{|c|}{ Products $^{a}$} \\
\hline & & & & 2 & 1 \\
\hline 1 & $17 \mathrm{eq}^{\mathrm{O}}$ & $\mathrm{K}_{2} \mathrm{CO}_{3}(17 \mathrm{eq})$ & $\alpha-\operatorname{cac}^{c}(1 \mathrm{eq})$ & $(+)$ & $(-)$ \\
\hline 2 & $17 e q$ & $\mathrm{~K}_{2} \mathrm{CO}_{3}(17 \mathrm{eq})$ & $\mathrm{ac}^{\mathrm{u}}$ (solvent) & - & - \\
\hline 3 & - & $\mathrm{NaH}$ (1eq) & $\operatorname{acp}^{t}(1 e q)$ & - & - \\
\hline 4 & - & $\mathrm{K}_{2} \mathrm{CO}_{3}(1 \mathrm{eq})$ & $\alpha-\operatorname{cac}(0.91$ eq $)$ & $(-)$ & $(+)$ \\
\hline
\end{tabular}

${ }^{\mathrm{a}}(+)$ denotes formation of the product, (-) denotes absence of the product, and "-" denotes product not expected. ${ }^{\text {b }}$ Respect to ADT$\mathrm{OH} .{ }^{c} \alpha$-cac: $\alpha$-chloroacetone. ${ }^{\mathrm{d}}$ ac: acetone. ${ }^{\mathrm{e}}$ acp: acetophenone
Secondly other enolate, phenacyl bromide, and other $3 \mathrm{H}$-1,2-dithiole-3-thione were studied (Table 2). The $3 \mathrm{H}$-1,2-dithiole-3-thione transformation to the corresponding (E)-3-[1-(alkylthio)alkylidene]-3H-1,2dithiole occurred in all the cases. Thirdly, microwave-irradiation heating was studied as alternative experimental conditions (Table 2).

Table 2. Scope of the transformation $3 H$-1,2-dithiole-3thione to $(E)-3-[1$-(alkylthio)alkylidene]-3H-1,2-dithiole.

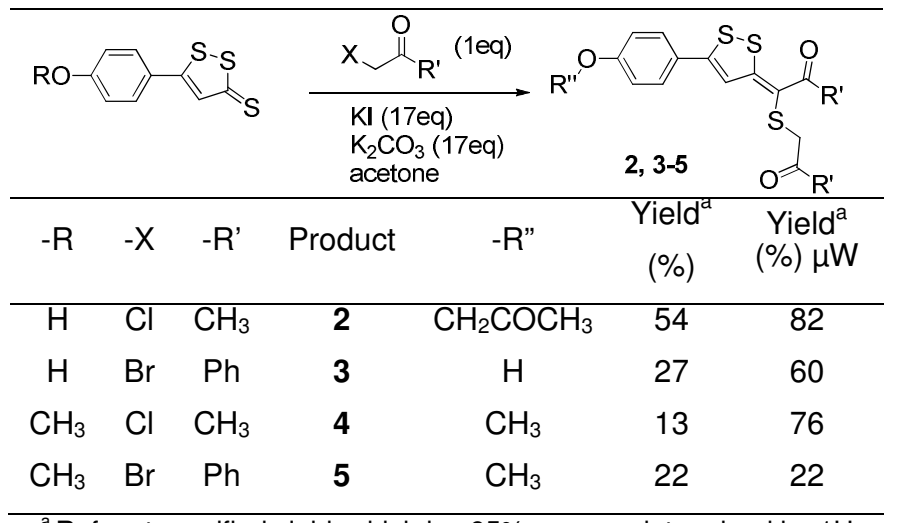

${ }^{a}$ Refers to purified yield, which is $>95 \%$ pure as determined by $1 \mathrm{H}$ NMR spectroscopy.

Additionally, we carried out a theoretical study explaining the unexpected reactivity in terms of Pearson hard and soft acid base (HSAB) theory.

\section{CONCLUSION}

The nucleophile of the following intermediate, result of the addition of iodine, could explain the observed product.

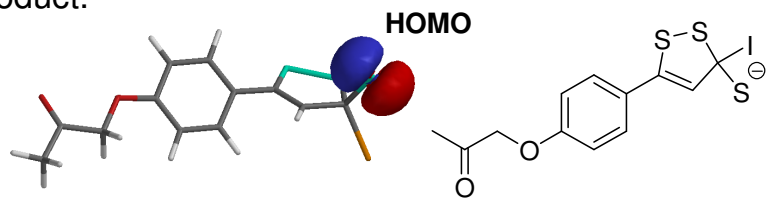

ACKNOWLEDGEMENTS

ANII and CSIC-UdelaR.

\section{REFERENCES}

${ }^{1}$ Félix, S.; Ascenso, J.R.; Lamartine, R.; Pereira, J.L.C. Tetrahedron, 1999, $55,8539-8546$ 